\title{
Free actions on products of spheres at high dimensions
}

\author{
OSMAN BERAT OKUTAN \\ ERGÜN YALÇIN
}

\begin{abstract}
A classical conjecture in transformation group theory states that if $G=(\mathbb{Z} / p)^{r}$ acts freely on a product of $k$ spheres $S^{n_{1}} \times \cdots \times S^{n_{k}}$, then $r \leq k$. We prove this conjecture in the case where the dimensions $\left\{n_{i}\right\}$ are high compared to all the differences $\left|n_{i}-n_{j}\right|$ between the dimensions.
\end{abstract}

57S25; $20 \mathrm{~J} 06$

\section{Introduction}

Let $G$ be a finite group. The rank of $G$, denoted by $\operatorname{rk}(G)$, is defined as the largest integer $s$ such that $(\mathbb{Z} / p)^{s} \leq G$ for some prime $p$. It is known that $G$ acts freely and cellularly on a finite complex homotopy equivalent to a sphere $S^{n}$ if and only if $\operatorname{rk}(G)=1$. This follows from the results due to P A Smith [12] and R Swan [13]. As a generalization of this, it has been conjectured by Benson and Carlson [3] that $\operatorname{rk}(G)=\operatorname{hrk}(G)$ where $\operatorname{hrk}(G)$ is defined as the smallest integer $k$ such that $G$ acts freely and cellularly on a finite $\mathrm{CW}$-complex homotopy equivalent to a product of $k$ spheres. This conjecture is often referred to as the rank conjecture. Note that one direction of the Benson-Carlson conjecture is the following statement:

Conjecture 1.1 Let $p$ be a prime. If $G=(\mathbb{Z} / p)^{r}$ acts freely and cellularly on a finite $C W$-complex $X$ homotopy equivalent to $S^{n_{1}} \times \cdots \times S^{n_{k}}$, then $r \leq k$.

This conjecture is a classical conjecture that was studied intensely through the 80's and it has been proven that the conjecture is true under some additional assumptions. For example it is known that when the dimensions of the spheres are all equal, ie, $n=n_{1}=\cdots=n_{k}$, then the conjecture is true for all primes $p$ and for all positive integers $n$ except when $p=2$ and $n=3,7$. This was proved by G Carlsson [6] in the case where the $G$-action on the homology of $X$ is trivial and the general case is due to Adem and Browder [2]. The $p=2$ and $n=1$ case was proven later by Yalçın [14]. More recently, B Hanke [10] proved that Conjecture 1.1 is true in the case where 
$p \geq 3 \operatorname{dim} X$, ie, when the prime $p$ is large compared to the dimension of the space. In this paper, we prove Conjecture 1.1 for the other extreme, ie, when the dimensions of the spheres are high compared to all the differences between the dimensions.

Theorem 1.2 Suppose $G=(\mathbb{Z} / p)^{r}$ for a prime $p$ and $k, l$ are positive integers. Then there is an integer $N$ that depends only on $k, l$ and $G$ such that if $G$ acts freely and cellularly on a finite dimensional $C W$-complex $X$ homotopy equivalent to $S^{n_{1}} \times \cdots \times S^{n_{k}}$ where $n_{i} \geq N$ and $\left|n_{i}-n_{j}\right| \leq l$ for all $i, j$, then $r \leq k$.

The proof follows from a theorem of Browder [4] that gives a restriction on the order of groups acting freely on a finite dimensional CW-complex in terms of homology groups of the complex. We also use a method of gluing homology groups at different dimensions, which we first saw in a paper by Habegger [9], and a crucial result on the exponents of cohomology groups of elementary abelian $p$-groups, which is due to Pakianathan [11].

At the end of the paper we also prove a generalization of Theorem 1.2 to non-free actions, which was suggested to us by A Adem.

The paper is organized as follows: In Section 2, we list some well-known results about hypercohomology and in Section 3, we introduce Habegger's theorem on gluing homology at different dimensions. In Section 4, we discuss the exponents of Tate cohomology groups and in Section 5, we prove Theorem 1.2, which is our main theorem.

\section{Tate hypercohomology}

Let $G$ be a finite group and $M$ be a $\mathbb{Z} G$-module. The Tate cohomology of $G$ with coefficients in $M$ is defined as follows:

$$
\widehat{H}^{i}(G, M):=H^{i}\left(\operatorname{Hom}_{G}\left(F_{*}, M\right)\right)
$$

for all $i \in \mathbb{Z}$, where $F_{*}$ is a complete $\mathbb{Z} G$-resolution of $\mathbb{Z}$ (see Brown [5, page 134]). We can generalize this and define Tate hypercohomology of $G$ with coefficients in a chain complex $C_{*}$ of $\mathbb{Z} G$-modules. To do this, we need to extend the contravariant functor $\operatorname{Hom}_{G}(-, M)$ to $\operatorname{Hom}_{G}\left(-, C_{*}\right)$. We will define it as in Brown [5, page 5], but instead of defining it as a chain complex, we consider it as a cochain complex.

Suppose $C_{*}$ and $D_{*}$ are chain complexes over $\mathbb{Z} G$ with differentials $\partial^{C}$ and $\partial^{D}$, respectively. For all $n \in \mathbb{Z}$, let $\operatorname{Hom}_{G}\left(C_{*}, D_{*}\right)^{n}$ denote the set of graded $G$-module homomorphisms of degree $-n$ and define the boundary map $\delta^{n}$ by

$$
\delta^{n}(f)=f \partial^{C}-(-1)^{n} \partial^{D} f .
$$


Note that $\operatorname{Hom}_{G}\left(-, C_{*}\right)$ (resp. $\left.\mathscr{H o m}_{G}\left(C_{*},-\right)\right)$ becomes a covariant (resp. contravariant) functor from the category of chain complexes of $\mathbb{Z} G$-modules to the category of cochain complexes of abelian groups. Also, if $C_{*}$ is a chain complex concentrated at 0 with $C_{0}=M$, then $\operatorname{Hom}_{G}\left(-, C_{*}\right)$ is naturally equivalent to the functor $\operatorname{Hom}_{G}(-, M)$. Now, we define the Tate hypercohomology of a finite group $G$ with coefficients in $C_{*}$ as

$$
\widehat{H}^{i}\left(G, C_{*}\right):=H^{i}\left(\operatorname{Hom}_{G}\left(F_{*}, C_{*}\right)\right)
$$

for all $i \in \mathbb{Z}$, where $F_{*}$ is a complete $\mathbb{Z} G$-resolution of $\mathbb{Z}$. We immediately have $\hat{H}^{i}\left(G, \Sigma C_{*}\right) \cong \hat{H}^{i+1}\left(G, C_{*}\right)$, where $\left(\Sigma C_{*}\right)_{i}=C_{i-1}$ for all $i$. Therefore, if $C_{*}$ is a chain complex concentrated at $n$, then $\hat{H}^{i}\left(G, C_{*}\right) \cong \hat{H}^{i+n}\left(G, C_{n}\right)$. Also note that given a short exact sequence of chain complexes

$$
0 \rightarrow C_{*} \rightarrow D_{*} \rightarrow E_{*} \rightarrow 0
$$

of $\mathbb{Z} G$-modules, there is a long exact sequence of the following form

$$
\cdots \rightarrow \hat{H}^{i}\left(G, C_{*}\right) \rightarrow \hat{H}^{i}\left(G, D_{*}\right) \rightarrow \hat{H}^{i}\left(G, E_{*}\right) \rightarrow \hat{H}^{i+1}\left(G, C_{*}\right) \rightarrow \cdots .
$$

An important property of Hom functor is that if $P_{*}$ is a chain complex of projective $\mathbb{Z} G$-modules and $f_{*}: C_{*} \rightarrow D_{*}$ a weak equivalence of nonnegative chain complexes of $\mathbb{Z} G$-modules, then $f_{*}: \operatorname{Hom}_{G}\left(P_{*}, C_{*}\right) \rightarrow \operatorname{Hom}_{G}\left(P_{*}, D_{*}\right)$ is also a weak equivalence (see Brown [5, page 29]). Actually, Brown proves this result by assuming $P_{*}$ is nonnegative and $C_{*}$ and $D_{*}$ are arbitrary, but the same proof remains true if we assume $P_{*}$ is arbitrary and $C_{*}$ and $D_{*}$ are nonnegative. Using this, we obtain the following proposition:

Proposition 2.1 If $C_{*}$ is a nonnegative chain complex of $\mathbb{Z} G$-modules with homology concentrated at dimension $n$ and $H_{n}\left(C_{*}\right)=M$, then $\hat{H}^{i}\left(G, C_{*}\right) \cong \hat{H}^{i+n}(G, M)$.

Proof Let $Z_{n}$ denote the group of $n$-cycles in $C_{*}$. We have the following weak equivalences:

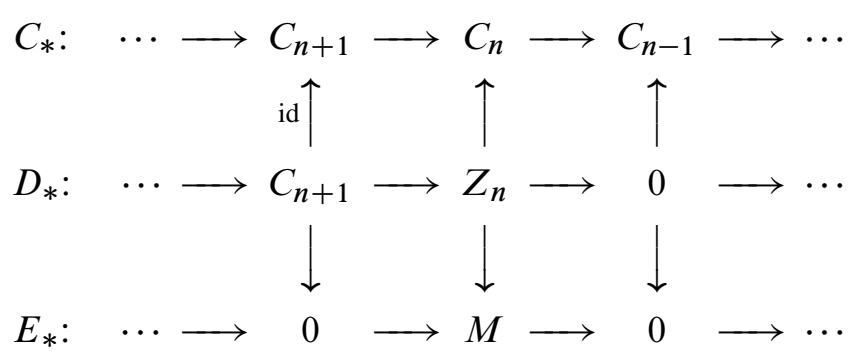

Therefore, $\hat{H}^{i}\left(G, C_{*}\right) \cong \hat{H}^{i}\left(G, D_{*}\right) \cong \hat{H}^{i}\left(G, E_{*}\right) \cong \hat{H}^{i+n}(G, M)$. 
An exact sequence

$$
K \stackrel{f}{\rightarrow} L \stackrel{g}{\rightarrow} M
$$

of $\mathbb{Z} G$-modules is called admissible if the inclusion map $\operatorname{im}(g) \hookrightarrow M$ is $\mathbb{Z}$-split (see Brown [5, page 129]). A $\mathbb{Z} G$-module $M$ is called relatively injective if $\operatorname{Hom}_{G}(-, M)$ takes an admissible exact sequence to an exact sequence of abelian groups. Projective $\mathbb{Z} G$-modules are relatively injective (see Brown [5, page 130]). Since a complete $\mathbb{Z} G$-resolution $F_{*}$ of $\mathbb{Z}$ is an exact sequence of free $\mathbb{Z} G$-modules, the sequence $F_{i+1} \rightarrow F_{i} \rightarrow F_{i-1}$ is admissible for all $i$. Hence if $P$ is a projective $\mathbb{Z} G$-module, then the Tate cohomology group $\hat{H}^{i}(G, P)=0$ for all $i$. This result generalizes to hypercohomology.

Proposition 2.2 If $P_{*}$ is a chain complex of projective $\mathbb{Z} G$-modules which has finite length, then $\hat{H}^{i}\left(G, P_{*}\right)=0$ for all $i$.

Proof Recall that we say a chain complex $C_{*}$ has finite length if there are integers $n$ and $m$ such that $C_{i}=0$ for all $i>n$ and $i<m$. By shifting $P_{*}$ if necessary, we can assume that $P_{*}$ is a finite dimensional nonnegative chain complex and prove the proposition by an easy induction on the dimension of $P_{*}$.

We say that two chain complexes $C_{*}$ and $D_{*}$ are freely equivalent if there is a sequence of chain complexes $C_{*}=E_{*}^{0}, \ldots, E_{*}^{n}=D_{*}$ such that either $E_{*}^{i}$ is an extension of $E_{*}^{i-1}$ or $E_{*}^{i-1}$ is an extension of $E_{*}^{i}$ by a finite length chain complex of free modules. Note that we say a chain complex $D_{*}$ is an extension of $C_{*}$ by a finite length chain complex of free modules if there is short exact sequence of chain complexes either of the form $0 \rightarrow C_{*} \rightarrow D_{*} \rightarrow F_{*} \rightarrow 0$ or of the form $0 \rightarrow F_{*} \rightarrow D_{*} \rightarrow C_{*} \rightarrow 0$, where $F_{*}$ is a finite length chain complex of free modules. As a corollary of Proposition 2.2, we have the following.

Corollary 2.3 If two chain complexes $C_{*}$ and $D_{*}$ are freely equivalent, then

$$
\hat{H}^{i}\left(G, C_{*}\right) \cong \hat{H}^{i}\left(G, D_{*}\right)
$$

for all $i$.

Before we conclude this section, we would like to note that there is a hypercohomology spectral sequence that converges to the Tate hypercohomology $\hat{H}^{*}\left(G, C_{*}\right)$ for a given chain complex $C_{*}$ of $\mathbb{Z} G$-modules. One way to obtain this spectral sequence is to consider the double complex $D^{p, q}=\operatorname{Hom}_{G}\left(F_{p}, C_{-q}\right)$ where the vertical and horizontal 
differentials are given by $\delta_{0}=\operatorname{Hom}_{G}(-, \partial)$ and $\delta_{1}=\operatorname{Hom}_{G}(\partial,-)$. Note that the total complex Tot $D^{*, *}$ with

$$
\operatorname{Tot}^{n} D^{*, *}=\bigoplus_{p+q=n} D^{p, q}
$$

and $\delta^{n}=\delta_{0}-(-1)^{n} \delta_{1}$ is a cochain complex homotopy equivalent to the cochain complex $\operatorname{Hom}_{G}\left(F_{*}, C_{*}\right)$. Filtering this double complex with respect to the index $p$ and then with respect to the index $q$, we obtain two spectral sequences

$$
\begin{aligned}
{ }^{\mathrm{I}} E_{2}^{p, q} & =\hat{H}^{p}\left(G, H_{-q}\left(C_{*}\right)\right) \Rightarrow \hat{H}^{p+q}\left(G, C_{*}\right), \\
{ }^{\mathrm{II}} E_{1}^{p, q} & =\widehat{H}^{q}\left(G, C_{-p}\right) \Rightarrow \widehat{H}^{p+q}\left(G, C_{*}\right) .
\end{aligned}
$$

Note that using these two spectral sequences it is possible to give alternative proofs for Propositions 2.1 and 2.2.

\section{Habegger's Theorem}

In [9, pages 433-434], Habegger uses a technique to "glue" homology groups of a chain complex at different dimensions. This technique will be crucial in the proof of Theorem 1.2, so we give a proof for it here. Before we state Habegger's Theorem, we recall the definition of syzygies of modules.

For every positive integer $n$, the $n^{\text {th }}$ syzygy of a $\mathbb{Z} G$-module $M$ is defined as the kernel of $\partial_{n-1}$ in a partial resolution of the form

$$
P_{n-1} \stackrel{\partial_{n-1}}{\longrightarrow} \cdots \rightarrow P_{1} \stackrel{\partial_{1}}{\longrightarrow} P_{0} \rightarrow M \rightarrow 0
$$

where $P_{0}, \ldots, P_{n-1}$ are projective $\mathbb{Z} G$-modules. We denote the $n^{\text {th }}$ syzygy of $M$ by $\Omega^{n} M$ and by convention we take $\Omega^{0} M=M$.

The $n^{\text {th }}$ syzygy of a module $M$ is well-defined only up to stable equivalence. Recall that two $\mathbb{Z} G$-modules $M$ and $N$ are called stably equivalent if there are projective $\mathbb{Z} G$-modules $P$ and $Q$ such that $M \oplus P \cong N \oplus Q$. Well-definedness of syzygies up to stable equivalence follows from a generalization of Schanuel's Lemma (see Brown [5, page 193]). Since for any two stably equivalent modules $M$ and $N$, we have $\hat{H}^{i}(G, M) \cong \hat{H}^{i}(G, N)$ for all $i$, we will ignore the fact that syzygies are welldefined only up to stable equivalence and treat $\Omega^{n} M$ as a unique module depending only on $M$ and $n$. Alternatively, one can fix a resolution for every $\mathbb{Z} G$-module $M$ and define $\Omega^{n} M$ as the kernel of $\partial_{n-1}$ in this unique resolution.

Theorem 3.1 (Habegger [9]) Let $C_{*}$ be a chain complex of $\mathbb{Z} G$-modules and $n, m$ are integers such that $m<n$. If $H_{k}\left(C_{*}\right)=0$ for all $k$ with $m<k<n$, then $C_{*}$ is freely equivalent to a chain complex $D_{*}$ such that: 
(i) $H_{i}\left(D_{*}\right)=H_{i}\left(C_{*}\right)$ for every $i \neq n, m$,

(ii) $H_{m}\left(D_{*}\right)=0$, and

(iii) there is an exact sequence of $\mathbb{Z} G$-modules

$$
0 \rightarrow H_{n}\left(C_{*}\right) \rightarrow H_{n}\left(D_{*}\right) \rightarrow \Omega^{n-m} H_{m}\left(C_{*}\right) \rightarrow 0 .
$$

Proof Let $F_{n-1} \rightarrow \cdots \rightarrow F_{m} \rightarrow H_{m}\left(C_{*}\right) \rightarrow 0$ be an exact sequence where the $F_{i}$ are free $\mathbb{Z} G$-modules. Consider the following diagram

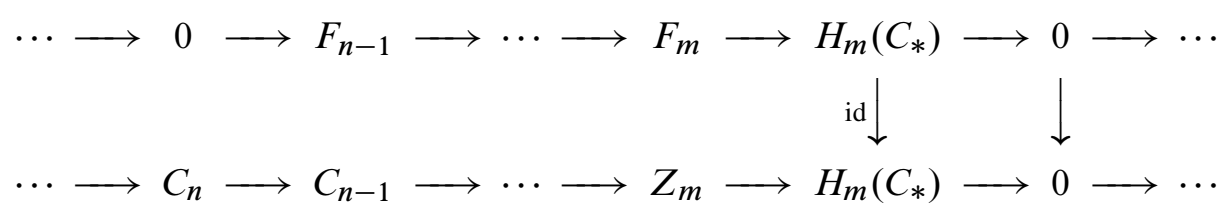

where $Z_{m}$ denotes the group of $m$-cycles in $C_{*}$. Since all $F_{i}$ are projective and the bottom row has no homology below dimension $n$, the identity map extends to a chain map between rows. Notice that this chain map gives a chain map $f_{*}: F_{*} \rightarrow C_{*}$ as follows

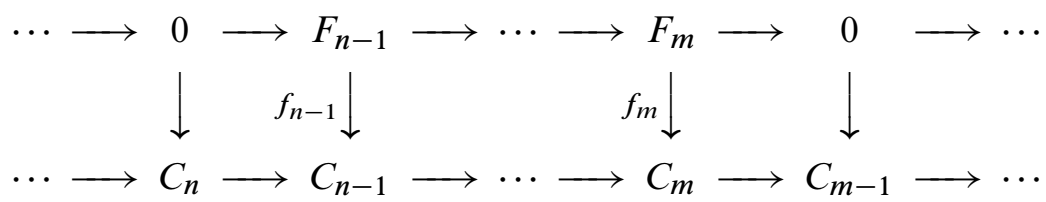

where the maps $f_{i}: F_{i} \rightarrow C_{i}$ for $i>m$ are the same as the maps in the first diagram above. The map $f_{m}: F_{m} \rightarrow C_{m}$ is defined as the composition

$$
F_{m} \stackrel{f_{m}^{\prime}}{\longrightarrow} Z_{m} \hookrightarrow C_{m}
$$

where $f_{m}^{\prime}: F_{m} \rightarrow Z_{m}$ is the map defined as the lifting of the identity map in the first diagram.

Now, let $D_{*}$ be the mapping cone of $f_{*}$. We have the following short exact sequence of the form

$$
0 \rightarrow C_{*} \rightarrow D_{*} \rightarrow \Sigma F_{*} \rightarrow 0
$$

so $C_{*}$ is freely equivalent to $D_{*}$. The corresponding long exact sequence of homology groups is

$$
\cdots \rightarrow H_{i}\left(F_{*}\right) \stackrel{f_{*}}{\rightarrow} H_{i}\left(C_{*}\right) \rightarrow H_{i}\left(D_{*}\right) \rightarrow H_{i-1}\left(F_{*}\right) \rightarrow \cdots .
$$

Assume first that $n>m+1$. Then $F_{*}$ has at least two terms and its homology is nonzero only at two dimensions $n-1$ and $m$. So, $H_{i}\left(C_{*}\right) \cong H_{i}\left(D_{*}\right)$ for all $i$ such that $i \neq m, m+1, n-1, n$. At dimension $m$, the map $f_{*}: H_{m}\left(F_{*}\right) \rightarrow H_{m}\left(C_{*}\right)$ is an 
isomorphism, so we get $H_{m}\left(D_{*}\right)=H_{m+1}\left(D_{*}\right)=0$. At dimension $n-1$, we have $H_{n-1}\left(C_{*}\right)=0$, so we get $H_{n-1}\left(D_{*}\right)=0$. We also have a short exact sequence of the form

$$
0 \rightarrow H_{n}\left(C_{*}\right) \rightarrow H_{n}\left(D_{*}\right) \rightarrow H_{n-1}\left(F_{*}\right) \rightarrow 0 .
$$

Since $H_{n-1}\left(F_{*}\right) \cong \Omega^{n-m}\left(H_{m}\left(C_{*}\right)\right)$, this gives the desired result.

If $n=m+1$, then $F_{*}$ has a single term $F_{m}$, so we have a sequence of the form

$$
0 \rightarrow H_{n}\left(C_{*}\right) \rightarrow H_{n}\left(D_{*}\right) \rightarrow F_{m} \stackrel{f_{*}}{\rightarrow} H_{m}\left(C_{*}\right) \rightarrow H_{m}\left(D_{*}\right) \rightarrow 0 .
$$

Since $f_{*}$ is surjective by construction, we conclude that $H_{m}\left(D_{*}\right)=0$ and there is a short exact sequence of the form

$$
0 \rightarrow H_{n}\left(C_{*}\right) \rightarrow H_{n}\left(D_{*}\right) \rightarrow \Omega^{1}\left(H_{m}\left(C_{*}\right)\right) \rightarrow 0
$$

as desired.

\section{Exponents of Tate cohomology groups}

To prove the main theorem, we need some results about the exponents of Tate cohomology groups. We first recall some definitions. The exponent of a finite abelian group $A$ is defined as the smallest positive integer $n$ such that $n a=0$ for all $a \in A$. We denote the exponent of $A$ by $\exp A$. Note that if $A \rightarrow B \rightarrow C$ is an exact sequence of finite abelian groups, then $\exp B$ divides $\exp A \cdot \exp C$. In this situation we sometimes write $\exp B / \exp A$ divides $\exp C$ to refer to the same fact even though $\exp B / \exp A$ may not be an integer in general.

The first result we prove is a proposition on the exponent of Tate cohomology group with coefficients in a filtered module. First let us explain the terminology that we will be using throughout the paper. Let $M$ be a $\mathbb{Z} G$-module and $A_{1}, A_{2}, \ldots, A_{n}$ be a sequence of $\mathbb{Z} G$-modules. If $M$ has a filtration $0=M_{0} \subseteq M_{1} \subseteq \cdots \subseteq M_{n}=M$ such that $M_{j} / M_{j-1} \cong A_{j}$ for all $j$, then we say $M$ has a filtration with sections $A_{1}-A_{2}-\cdots-A_{n}$.

Proposition 4.1 Let $M$ be a $\mathbb{Z} G$-module which has a filtration with sections $A_{1}-$ $A_{2}-\cdots-A_{n}$. Then, $\exp \widehat{H}^{i}(G, M)$ divides $\prod_{j=1}^{n} \exp \hat{H}^{i}\left(G, A_{j}\right)$.

Proof Let $0=M_{0} \subseteq M_{1} \subseteq \cdots \subseteq M_{n}=M$ be the filtration of $M$ with the sections as above. Then for every $j$, we have an exact sequence of $\mathbb{Z} G$-modules

$$
0 \rightarrow M_{j-1} \rightarrow M_{j} \rightarrow A_{j} \rightarrow 0,
$$


which gives a long exact Tate cohomology sequence of the following form:

$$
\cdots \rightarrow \hat{H}^{i}\left(G, M_{j-1}\right) \rightarrow \hat{H}^{i}\left(G, M_{j}\right) \rightarrow \hat{H}^{i}\left(G, A_{j}\right) \rightarrow \cdots .
$$

From this we observe that

$$
\exp \hat{H}^{i}\left(G, M_{j}\right) / \exp \hat{H}^{i}\left(G, M_{j-1}\right)
$$

divides the exponent of $\hat{H}^{i}\left(G, A_{j}\right)$. Multiplying these relations through all $j=$ $1, \ldots, n$, we get $\exp \hat{H}^{i}(G, M)$ divides $\prod_{j=1}^{n} \exp \hat{H}^{i}\left(G, A_{j}\right)$.

In [4], Browder proves a theorem that gives an upper bound on the order of a finite group $G$ in terms of the exponents of cohomology groups with coefficients in homology groups of a CW-complex on which $G$ acts freely. Since we use this theorem in the proof of our main theorem, we state it below and give a proof for it. The proof we give here is slightly different than the original proof. It uses Theorem 3.1 and Proposition 4.1.

Theorem 4.2 (Browder [4]) Let $C_{*}$ be a nonnegative, free, connected chain complex of dimension $n$. Then $|G|$ divides $\prod_{j=1}^{n} \exp H^{j+1}\left(G, H_{j}\left(C_{*}\right)\right)$.

Proof Let us take $C_{*}^{(0)}=C_{*}$ and for $j=1$ to $n$, define $C_{*}^{(j)}$ to be the chain complex obtained from $C_{*}^{(j-1)}$ by applying the method in Theorem 3.1 for the dimensions $n-j$ and $n$. Since $C_{*}$ is a finite dimensional chain complex of free $\mathbb{Z} G$-modules, by Proposition 2.2, $\hat{H}^{i}\left(G, C_{*}\right)=0$ for all $i$. Hence by Corollary 2.3 and Theorem 3.1, we have $\hat{H}^{i}\left(G, C_{*}^{(j)}\right)=0$ for all $i, j$. Notice that $C_{*}^{(n)}$ is a chain complex with homology concentrated at $n$. Let us denote the homology of $C_{*}^{(n)}$ at $n$ by $M$. Hence, by Proposition 2.1, we have $\hat{H}^{i}(G, M)=0$ for all $i$. By Theorem 3.1, $M$ has a filtration

$$
0 \subseteq H_{n}\left(C_{*}^{(0)}\right) \subseteq \cdots \subseteq H_{n}\left(C_{*}^{(n-1)}\right) \subseteq H_{n}\left(C_{*}^{(n)}\right)=M
$$

with sections $H_{n}\left(C_{*}\right)-\Omega^{1} H_{n-1}\left(C_{*}\right)-\cdots-\Omega^{n-1} H_{1}\left(C_{*}\right)-\Omega^{n} H_{0}\left(C_{*}\right)$. If we let $M^{\prime}:=H_{n}\left(C_{*}^{(n-1)}\right)$, then $M^{\prime}$ has a filtration with sections

$$
H_{n}\left(C_{*}\right)-\Omega^{1} H_{n-1}\left(C_{*}\right)-\cdots-\Omega^{n-1} H_{1}\left(C_{*}\right)
$$

and there is a short exact sequence of the form

$$
0 \rightarrow M^{\prime} \rightarrow M \stackrel{\pi}{\rightarrow} \Omega^{n} H_{0}\left(C_{*}\right) \rightarrow 0 .
$$

Note that $H_{0}\left(C_{*}\right) \cong \mathbb{Z}$, so we obtain an exact sequence of the form

$$
\cdots \rightarrow \widehat{H}^{n}(G, M) \stackrel{\pi_{*}}{\rightarrow} \hat{H}^{n}\left(G, \Omega^{n} \mathbb{Z}\right) \rightarrow \hat{H}^{n+1}\left(G, M^{\prime}\right) \rightarrow \hat{H}^{n+1}(G, M) \rightarrow \cdots .
$$


Since $\hat{H}^{i}(G, M)=0$ for all $i$, we obtain

$$
\widehat{H}^{n+1}\left(G, M^{\prime}\right) \cong \hat{H}^{n}\left(G, \Omega^{n} \mathbb{Z}\right) \cong \hat{H}^{0}(G, \mathbb{Z}) \cong \mathbb{Z} /|G| .
$$

Hence by Proposition 4.1, we get $|G|=\exp \hat{H}^{n+1}\left(G, M^{\prime}\right)$ divides the product

$$
\prod_{j=1}^{n} \exp \hat{H}^{n+1}\left(G, \Omega^{n-j} H_{j}\left(C_{*}\right)\right) \text {. }
$$

Since $\hat{H}^{n+1}\left(G, \Omega^{n-j} H_{j}\left(C_{*}\right)\right) \cong H^{j+1}\left(G, H_{j}\left(C_{*}\right)\right)$, this gives the desired result.

As a corollary of Theorem 4.2, Browder gives a proof for a theorem of G Carlsson [6] that says that if $G=(\mathbb{Z} / p)^{r}$ acts freely on a finite dimensional CW-complex $X \simeq\left(S^{n}\right)^{k}$ with trivial action on homology, then $r \leq k$. The main observation is that when $G=(\mathbb{Z} / p)^{r}$ and $M$ is a trivial $\mathbb{Z} G$-module, the exponent of $H^{i}(G, M)$ divides $p$ for all $i \geq 1$. This follows easily by induction on $r$ using properties of the transfer map in group cohomology. So, from the relation given in Theorem 4.2, one obtains that if $G$ acts freely on a finite dimensional CW-complex $X \simeq\left(S^{n}\right)^{k}$ with trivial action on homology, then $|G|=p^{r}$ divides $p^{k}$, which gives $r \leq k$.

Note that the assumption that $G$ acts trivially on the homology of $X$ is crucial in the above argument since for an arbitrary $\mathbb{Z} G$-module, the exponent of $H^{i}(G, M)$ can be as large as the order of $|G|$. In fact, if we take $M=\Omega^{i}(\mathbb{Z})$ for some positive integer $i$, then we have $H^{i}(G, M) \cong \mathbb{Z} /|G|$, so the exponent of $H^{i}(G, M)$ is equal to $|G|$ in this case. Taking the direct sum of all such modules over all $i$, one can obtain a $\mathbb{Z} G$-module $M$ such that the exponent of $H^{i}(G, M)$ is equal to $|G|$ for every $i \geq 0$. The following theorem says that when $M$ is finitely generated this situation cannot happen and that the exponent of $H^{i}(G, M)$ eventually becomes small at high dimensions.

Theorem 4.3 (Pakianathan [11]) Let $G=(\mathbb{Z} / p)^{r}$ and $M$ be a finitely generated $\mathbb{Z} G$-module. Then, there is an integer $N$ such that the exponent of $H^{i}(G, M)$ divides $p$ for all $i \geq N$.

Proof By Theorem 7.4.1 in Evens [8, page 87], $H^{*}(G, M)$ is a finitely generated module over the ring $H^{*}(G, \mathbb{Z})$. Let $u_{1}, \ldots, u_{k}$ be homogeneous elements generating $H^{*}(G, M)$ as an $H^{*}(G, \mathbb{Z})$-module and let $N=1+\max _{j}\left\{\operatorname{deg} u_{j}\right\}$. If $u \in H^{i}(G, M)$ such that $i \geq N$, then we can write $u=\sum_{j=1}^{k} \alpha_{j} u_{j}$ for some homogeneous elements $\alpha_{j}$ in $H^{*}(G, \mathbb{Z})$ with $\operatorname{deg} \alpha_{j} \geq 1$ for all $j$. Since $\exp H^{i}(G, \mathbb{Z})$ divides $p$ for all $i \geq 1$, we have $p \alpha_{j}=0$ for all $j$. Hence we obtain $p u=\sum_{j=1}^{k} p \alpha_{j} u_{j}=0$ as desired. 


\section{Proof of the main theorem}

Let $G=(\mathbb{Z} / p)^{r}$ and $k, l$ be positive integers. We will show that there is an integer $N$ such that if $G$ acts freely and cellularly on a CW-complex $X$ homotopy equivalent to $S^{n_{1}} \times \cdots \times S^{n_{k}}$ where $\left|n_{i}-n_{j}\right| \leq l$ and $n_{i} \geq N$ for all $i, j$, then $r \leq k$.

Suppose that $G$ acts freely and cellularly on some CW-complex $X$ homotopy equivalent to $S^{n_{1}} \times \cdots \times S^{n_{k}}$ where $\left|n_{i}-n_{j}\right| \leq l$ for all $i, j$. Let $n=\max \left\{n_{i} \mid i=1, \ldots, k\right\}$ and let $a_{i}=n-n_{i}$ for all $i$. Consider the cellular chain complex $C_{*}(X)$ of the CWcomplex $X$. The complex $C_{*}(X)$ is a nonnegative, connected, and finite-dimensional chain complex of free $\mathbb{Z} G$-modules and has nonzero homology only at the following dimensions other than dimension zero:

$$
\begin{array}{cc}
\text { (1) } & n-a_{1}, n-a_{2}, \ldots, n-a_{k} \\
\text { (2) } & 2 n-a_{1}-a_{2}, 2 n-a_{1}-a_{3}, \ldots, 2 n-a_{k-1}-a_{k} \\
& \vdots \\
\text { (j) } & j n-\left(a_{1}+\cdots+a_{j}\right), \ldots, j n-\left(a_{k-j+1}+\cdots+a_{k}\right) \\
& \vdots \\
\text { (k) } & k n-\left(a_{1}+a_{2}+\cdots+a_{k}\right)
\end{array}
$$

If $n>l k$, then we have $n>a_{1}+\cdots+a_{k}$, which implies that for all $j$, the dimensions listed on the $j^{\text {th }}$ row are strictly larger than the dimensions listed on the $(j-1)^{\text {st }}$ row. Since this fact is crucial for our argument, we will assume that the integer $N$ in the statement of the theorem satisfies $N>l k$ to guarantee that this condition holds.

Now we can apply Habegger's argument given in Theorem 3.1 to glue all the homology groups at the dimensions listed on the $j^{\text {th }}$ row above to the homology at dimension $j n$ for all $j=1, \ldots, k$. The resulting complex $D_{*}$ is a connected, finite-dimensional chain complex of free $\mathbb{Z} G$-modules that has homology only at dimensions $0, n, 2 n, \ldots, k n$. Let $M_{j}:=H_{j n}\left(D_{*}\right)$ for all $j=1, \ldots, k$. Note that by construction $M_{j}$ is a finitely generated $\mathbb{Z} G$-module for all $j$ since syzygies of finitely generated $\mathbb{Z} G$-modules are finitely generated when $G$ is a finite group.

Now we can apply Theorem 4.3 to find an integer $N_{j}$ for each $j$ such that if $i \geq N_{j}$, then $\exp H^{i}\left(G, M_{j}\right)$ divides $p$. Suppose that for a fixed $G=(\mathbb{Z} / p)^{r}, k$ and $l$, there are only finitely many possibilities for $\mathbb{Z} G$-modules $M_{j}$ up to stable equivalence. Then by taking the maximum of the $N_{j}$ over all possible $M_{j}$, we can find an integer $N_{j}^{\max }$ for each $j$ such that if $i \geq N_{j}^{\max }$, then $\exp H^{i}\left(G, M_{j}\right)$ divides $p$ for all possible $M_{j}$ that may occur. Then we can take $N=\max _{j} N_{j}^{\max }$ and complete the proof in the 
following way. By Theorem 4.2, we have $|G|=p^{r}$ divides

$$
\prod_{j=1}^{k} H^{j n+1}\left(G, H_{j n}\left(D_{*}\right)\right)=\prod_{j=1}^{k} H^{j n+1}\left(G, M_{j}\right) .
$$

So, if $n \geq N$, then $p^{r}$ divides $p^{k}$, which gives $r \leq k$ as desired.

Hence to complete the proof, it only remains to show that for fixed $G=(\mathbb{Z} / p)^{r}, k$ and $l$, there are only finitely many possibilities for $\mathbb{Z} G$-modules $M_{j}$ up to stable equivalence. To show this, first note that for a fixed $l$, there are finitely many $k$-tuples $\left(a_{1}, \ldots, a_{k}\right)$ with the property that $0 \leq a_{i} \leq l$ for all $i$. So we can assume that we have a fixed $k$-tuple $\left(a_{1}, \ldots, a_{k}\right)$. Let us also fix an integer $j$ and show there are only finitely many possibilities for $M_{j}=H_{j n}\left(D_{*}\right)$.

Let $s_{1}<\cdots<s_{m}$ be a sequence of integers such that $\left\{j n-s_{1}, \ldots, j n-s_{m}\right\}$ is the set of all distinct dimensions on the $j^{\text {th }}$ row of the above diagram. Note that the complex $D_{*}$ is constructed with the repeated usage of Theorem 3.1, so the module $M_{j}=H_{j n}\left(D_{*}\right)$ has a filtration

$$
0=K_{0} \subseteq K_{1} \subseteq \cdots \subseteq K_{m}=M_{j}
$$

such that $K_{i} / K_{i-1} \cong \Omega^{s_{i}}\left(A_{i}\right)$ where $A_{i}=H_{j n-s_{i}}(X)$. For all $i$, the module $A_{i}$ is a $\mathbb{Z}$-free $\mathbb{Z} G$-module with $\mathbb{Z}$-rank less than or equal to $\left(\begin{array}{c}k \\ j\end{array}\right)$, so by the JordanZassenhaus Theorem (see Corollary 79.12 in Curtis and Reiner [7, page 563]), there are only finitely many possibilities for $A_{i}$ up to isomorphism.

We will inductively show that there exist only finitely many possibilities for $K_{i}$ up to stable equivalence. For $i=1$, we have $K_{1}=\Omega^{s_{1}}\left(A_{1}\right)$ so this follows from the fact that there are only finitely many possibilities for $A_{1}$ and that syzygies are well-defined up to stable equivalence. For $i>1$, consider the following short exact sequence:

$$
0 \rightarrow K_{i-1} \rightarrow K_{i} \rightarrow \Omega^{s_{i}} A_{i} \rightarrow 0
$$

By induction we know that there are only a finite number of possibilities for $K_{i-1}$ up to stable equivalence. By a similar argument as above, the same is true for $\Omega^{s_{i}}\left(A_{i}\right)$. The extensions like the ones above are classified by the ext-group $\operatorname{Ext}_{\mathbb{Z} G}^{1}\left(\Omega^{s_{i}}\left(A_{i}\right), K_{i-1}\right)$ and since both modules are $\mathbb{Z}$-free, these ext-groups are well-defined up to stable equivalence. So, it remains to show that

$$
\operatorname{Ext}_{\mathbb{Z} G}^{1}\left(\Omega^{s_{i}}\left(A_{i}\right), K_{i-1}\right)=\operatorname{Ext}_{\mathbb{Z} G}^{s_{i}+1}\left(A_{i}, K_{i-1}\right)
$$

is a finite group. Note that since both $A_{i}$ and $K_{i-1}$ are finitely generated,

$$
\operatorname{Ext}_{\mathbb{Z} G}^{s_{i}+1}\left(A_{i}, K_{i-1}\right)
$$


is a finitely generated abelian group. Moreover, since $A_{i}$ is $\mathbb{Z}$-free, it has an exponent divisible by $|G|$. So, $\operatorname{Ext}_{\mathbb{Z} G}^{s_{i}+1}\left(A_{i}, K_{i-1}\right)$ is a finite group. This completes the proof of Theorem 1.2.

We conclude this section with a generalization of Theorem 1.2 to non-free actions. The exact statement is as follows.

Theorem 5.1 Suppose $G=(\mathbb{Z} / p)^{r}$ for a prime $p$ and $k, l$ are positive integers. Then there is an integer $N$ that depends only on $k, l$ and $G$ such that if $G$ acts cellularly on a finite dimensional $C W$-complex $X$ homotopy equivalent to $S^{n_{1}} \times \cdots \times S^{n_{k}}$ where $n_{i} \geq N$ and $\left|n_{i}-n_{j}\right| \leq l$ for all $i, j$, then $r-s \leq k$ where $s$ is the largest integer such that $\left|G_{X}\right|=p^{s}$ for some $x \in X$.

Proof Let $C_{*}:=C_{*}(X)$ denote the cellular chain complex of $X$ and let $\varepsilon: C_{*} \rightarrow \mathbb{Z}$ be the map induced by the constant map $X \rightarrow p t$. The arguments in the proof of Theorem 1.2 can be repeated to prove that there is an integer $N$ such that if $n_{i} \geq N$ and $\left|n_{i}-n_{j}\right| \leq l$ for all $i, j$, then

$$
p^{k} \widehat{H}^{0}(G, \mathbb{Z}) \subseteq \operatorname{im}\left\{\varepsilon_{*}: \widehat{H}^{0}\left(G, C_{*}\right) \rightarrow \widehat{H}^{0}(G, \mathbb{Z})\right\} .
$$

This can be seen by a spectral sequence argument or by the filtration argument given in the proof of Theorem 4.2. To see it using the filtration argument, observe that the map $\epsilon_{*}$ can be written as a composition

$$
\epsilon_{*}: \hat{H}^{0}\left(G, C_{*}\right) \cong \hat{H}^{0}\left(G, C_{*}^{(n)}\right) \cong H^{n}(G, M) \stackrel{\pi_{*}}{\longrightarrow} \hat{H}^{n}\left(G, \Omega^{n} \mathbb{Z}\right) \cong \hat{H}^{0}(G, \mathbb{Z})
$$

where the module $M$ and the map $\pi_{*}$ are as given in the proof of Theorem 4.2. Repeating the arguments in the proof of Theorem 1.2, we can show that there is an integer $N$ such that if $n_{i} \geq N$ and $\left|n_{i}-n_{j}\right| \leq l$ for all $i, j$, then $\exp \hat{H}^{n+1}\left(G, M^{\prime}\right)$ divides $p^{k}$ where $M^{\prime}$ is as in the proof of Theorem 4.2. Using the long exact sequence given in the proof of Theorem 4.2, we can conclude that $p^{k} \widehat{H}^{0}(G, \mathbb{Z}) \subseteq \operatorname{im} \varepsilon_{*}$.

The inclusion given above implies that $|G|=p^{r}$ divides $p^{k} \cdot \exp \hat{H}^{0}\left(G, C_{*}\right)$. Hence the proof will be complete if we can show that $\exp \hat{H}^{0}\left(G, C_{*}\right)$ divides $p^{s}$ where $s$ is the largest integer such that $\left|G_{x}\right|=p^{s}$ for some $x \in X$. However this is already known to be true as proven by A Adem [1, Theorems 3.1 and 3.2]. So the proof is complete.

\section{Acknowledgements}

We thank Jonathan Pakianathan and Alejandro Adem for many helpful conversations on the paper and the referee for a careful reading of the paper and for his/her corrections and helpful comments. The second author is partially supported by TÜBITAKTBAG/110T712. 


\section{References}

[1] A Adem, Torsion in equivariant cohomology, Comment. Math. Helv. 64 (1989) 401411 MR998857

[2] A Adem, W Browder, The free rank of symmetry of $\left(S^{n}\right)^{k}$, Invent. Math. 92 (1988) 431-440 MR936091

[3] D J Benson, J F Carlson, Complexity and multiple complexes, Math. Z. 195 (1987) 221-238 MR892053

[4] W Browder, Cohomology and group actions, Invent. Math. 71 (1983) 599-607 MR695909

[5] K S Brown, Cohomology of groups, Graduate Texts in Mathematics 87, Springer, New York (1982) MR672956

[6] G Carlsson, On the rank of abelian groups acting freely on $\left(S^{n}\right)^{k}$, Invent. Math. 69 (1982) 393-400 MR679764

[7] C W Curtis, I Reiner, Representation theory of finite groups and associative algebras, Pure and Applied Mathematics 11, Interscience Publishers, a division of John Wiley \& Sons, New York (1962) MR0144979

[8] L Evens, The cohomology of groups, Oxford Mathematical Monographs, The Clarendon Press, New York (1991) MR1144017

[9] N Habegger, Hypercohomology varieties for complexes of modules, the realizability criterion, and equivalent formulations of a conjecture of Carlsson, from: "The Arcata conference on representations of finite groups", (P Fong, editor), Proc. Sympos. Pure Math. 47, Amer. Math. Soc. (1987) 431-437 MR933380

[10] B Hanke, The stable free rank of symmetry of products of spheres, Invent. Math. 178 (2009) 265-298 MR2545682

[11] J Pakianathan, private communication (2012)

[12] P A Smith, Permutable periodic transformations, Proc. Nat. Acad. Sci. U. S. A. 30 (1944) 105-108 MR0010278

[13] R G Swan, Periodic resolutions for finite groups, Ann. of Math. 72 (1960) 267-291 MR0124895

[14] E Yalçın, Group actions and group extensions, Trans. Amer. Math. Soc. 352 (2000) 2689-2700 MR1661282

Department of Mathematics, The Ohio State University, Columbus, OH 43210-1174, USA Department of Mathematics, Bilkent University, 06800 Ankara, Turkey okutan.1@osu.edu, yalcine@fen.bilkent.edu.tr http://www.math.osu.edu/people/okutan.1, http://www.fen.bilkent.edu.tr/ yalcine/

Received: 17 October 2012 Revised: 13 February 2013 